\title{
Iris Pattern Recognition using Support Vector Machines and Artificial Neural Networks
}

\author{
Asim Ali Khan ${ }^{1}$, Smriti Kumar², Mister Khan ${ }^{3}$ \\ Associate Professor, Electrical and Instrumentation Deptt, Sant Longowal Institute of Engineering and Technology, \\ Longowal, India ${ }^{1}$ \\ Research Scholar, Electrical and Instrumentation Deptt, Sant Longowal Institute of Engineering and Technology, \\ Longowal, India ${ }^{2,3}$
}

\begin{abstract}
An authentic personal identification infrastructure is required to control the access in order to secure areas or materials. Biometric technology is based on physiological or behavioral characteristics of a human body. Iris recognition system consists of image acquisition, localization, normalization, features extraction and encoding, and classification. Iris images are downloaded from CASIA Iris V1.0 database for study. To separate the iris region from the eye image, Hough transform is used. Circular Hough transform is used to localize circular iris and pupil region while parabolic Hough transform is used to enhance the occluding eyelids and eyelashes. Daugman's rubber sheet model is used to normalize the extracted iris region into a rectangular block with constant polar dimensions. After normalization, 2D Gabor filter is employed to extract the important features from iris. Iris provides texture information which is unique, universal and contains high randomness. Feature extraction is performed by convolving the normalized iris region with 2D Gabor filter which gives the phase information. The phase data represented by a data set is utilized as input for classifiers. The classifiers used in this study are Artificial Neural Networks (ANN) and Support Vector Machines (SVM). This study shows that Support Vector Machines gives higher recognition rate than Artificial Neural Networks.
\end{abstract}

Keywords: Iris segmentation, Iris recognition, Hough transform, 2D Gabor filter, Support Vector Machines, Artificial Neural Networks.

\section{INTRODUCTION}

With the fast developing trends in Information The framework of iris recognition system is shown in Fig. Technology, the identity frauds in our society are also 1 . Iris recognition system consists of iris image reaching unprecedented proportion and therefore there is acquisition, segmentation, feature extraction and an increasing requirement for Information and Network recognition of the iris region using classifiers in a digital Security, which demands for efficient and automatic eye image. Iris image segmentation includes eye image personal identification system. Identification of people is filtering, iris localization and iris normalization. Each of getting more and more importance in the increasing these uses various algorithms.

networked society ${ }^{[1]}$. Traditional password based system like password, PIN, keys are not much safer because it can be lost or stolen and cannot differentiate between authorized and imposter. Biometrics refers to identifying an individual based on his or her distinguish characteristics ${ }^{[2]}$. Biometrics enlists physiological, behavioral or biological characteristics for accurate identification of an individual. Various biometric traits include fingerprint, hand geometry, iris, face, retina, Gait (way of walk), keystroke dynamics, palm print, signature, voice, DNA, ear ${ }^{[3]}$. For identification, the iris recognition is most suitable and accurate biometric technology because of its uniqueness and stability. The human iris is an annular part between pupil and white sclera. Iris region is sometimes called iris texture. The iris texture provides many minute characteristics such as freckles, coronas, stripes, furrows, crypts etc. ${ }^{[4]}$. The development of iris takes two years and then pattern of iris became constant over person's life time. Not only the irises of identical twins are different but also left and right irises of the same person are different.

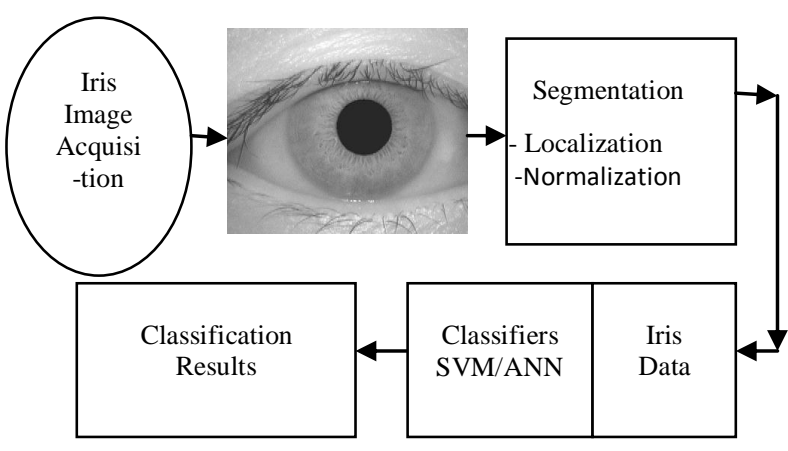

Fig. 1. The Architecture of Iris Recognition System

\section{IRIS IMAGE ACQUISITION}

Image acquisition means to capture the human's eye region image. The captured image must be of high quality to work properly for iris recognition system. The acquired image of eye must have good resolution and clarity for supporting the better recognition. The illumination in the image must be removed because illumination will results 
in poor quality images with lots of reflection. For capturing good quality images with high resolution and low illumination, infra red camera should be used.

\section{III.IRIS IMAGE SEGMENTATION}

Before segmentation, the low pass Gaussian filter is used for smoothening and sharpening the eye image. The illumination effect will also be removed by low pass Gaussian filter. The acquired image also contains irrelevant parts like eyelids, eyelashes, should be removed. Before extracting the features from the original eye image, eye image should be preprocessed to localize pupil and iris boundaries and normalize the iris ${ }^{[5]}$.

\section{A. Iris Localization}

For recognition purpose, the iris part should be separated from the eye image. The iris is a ring shaped part in eye with pupil as inner boundary and sclera as outer boundary. Both the boundaries can approximately take as inner and outer circles of iris. For detecting the pupil and iris boundary, circular Hough transform ${ }^{[6]}$ is used. Canny edge detector is employing to generate an edge map, which finds edges where the grayscale intensity of the image changes the most. These areas are founded by determining gradients of the image ${ }^{[5]}$. In order to make the circle detection process more efficient and accurate, the Hough transform for the iris/sclera boundary was performed first, then the Hough transform for the iris/pupil boundary was performed within the iris region, instead of the whole eye region, since the pupil is always within the iris region. After this process was complete, six parameters are stored, the radius and centre coordinates for both circles ${ }^{[7]}$ by the following equation.

$$
\mathrm{x}_{\mathrm{c}}{ }^{2}+\mathrm{y}_{\mathrm{c}}{ }^{2}-\mathrm{r}^{2}=0
$$

where $x_{c}, y_{c}$ are centre coordinates and $r$ is radius of a circle.

The inner and outer boundaries of iris are shown in Fig. 2.

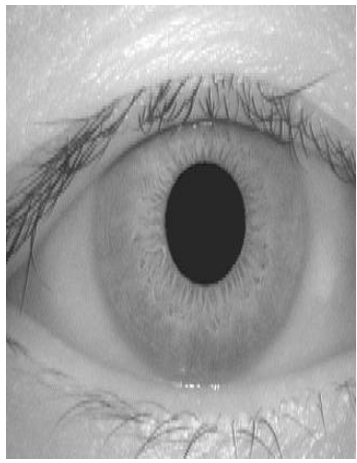

(a) Original image

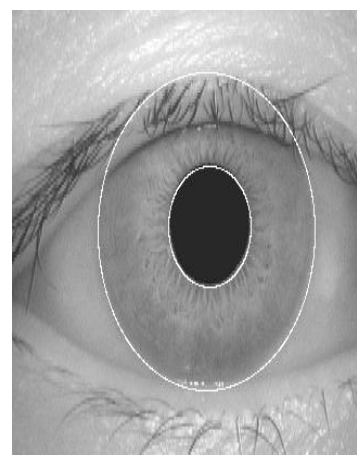

(b) Localized image
Fig. 2. Localization of the iris boundaries

Eyelids were isolated by first fitting a line to the upper and lower eyelids using the linear Hough transform. A second horizontal line is then drawn, which intersects with the first line at the iris edge that is closest to the pupil. This process is done for both upper and lower eyelids. The second horizontal line allows maximum isolation of eyelid regions.

\section{B. Iris Localization}

People have different sizes of iris. So, after iris localization process, the next stage is to transform the iris region so that it has fixed dimensions in order to allow comparisons. Factors which affect size of iris are intraclass variability i.e. illumination, varying of pose, noise and pollution, and inter-class variability i.e. person to person. During normalization, iris and pupil are nonconcentric ${ }^{[8,9]}$. These factors affect the result of iris matching. To get accurate result, localized iris is transformed from Cartesian to polar coordinate system. For transformation, Daugman's Rubber sheet model ${ }^{[8]}$ is followed. The homogenous rubber sheet model remaps each point within the iris region to a pair of polar coordinates $(r, \theta)$ where $r$ is on the interval $[0,1]$ and $\theta$ is angle $[0,2 \Pi]$ as shown in Fig. 3.

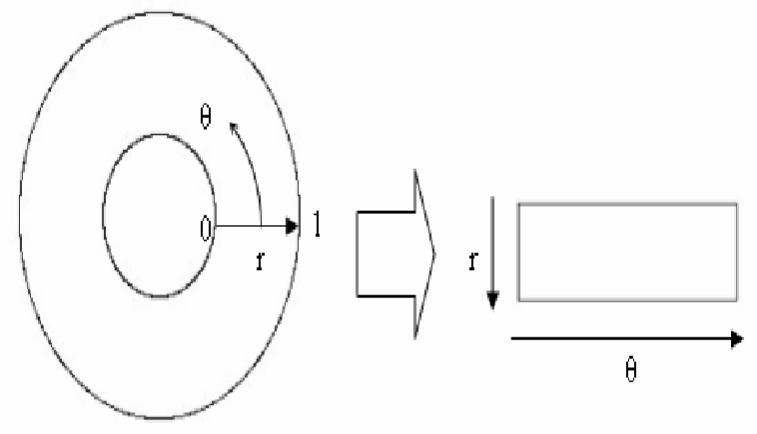

Fig. 3. Daugman rubber sheet model

The remapping of the iris region from $(x, y)$ Cartesian coordinates to the normalized non-concentric polar representation is modeled as

$$
\mathrm{I}(\mathrm{x}(\mathrm{r}, \Theta), \mathrm{y}(\mathrm{r}, \Theta)) \longrightarrow \mathrm{I}(\mathrm{r}, \Theta)
$$

With $x(r, \Theta)=(1-r) x_{p}(\Theta)+r_{1}(\Theta)$

$$
y(r, \theta)=(1-r) y_{p}(\Theta)+r y_{l}(\Theta)
$$

where $\mathrm{I}(\mathrm{x}, \mathrm{y})$ is the iris region image, $(\mathrm{x}, \mathrm{y})$ are the original Cartesian coordinates, ( $r, \Theta)$ are the corresponding normalized polar coordinates, and $\mathrm{x}_{\mathrm{p}}, \mathrm{y}_{\mathrm{p}}$ and $\mathrm{x}_{\mathrm{l}}, \mathrm{y}_{\mathrm{l}}$ are the coordinates of the pupil and iris boundaries along the $\Theta$ direction. In order to prevent non-iris region data from corrupting the normalized representation, data points which occur along the pupil border or the iris border are discarded as in Fig.4.

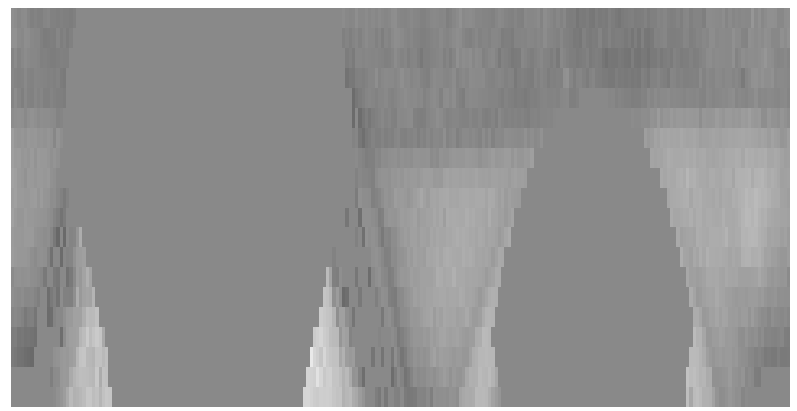

Fig. 4. Iris Normalization 


\section{IV.FEATURE EXTRACTION/ENCODING}

The iris has various structures which provide texture information. To authentic the individuals, the most distinguishing features that present in the region is extracted ${ }^{[7,10]}$. Various methods for feature extraction are Wavelet encoding, Gabor filter, Log-Gabor filter, Haar wavelet, 2D Gabor filter, Laplacian of Gaussian filters and zero crossings of the 1D wavelet. In the present work, 2D Gabor filter is used. The functional form of 2D Gabor filters ${ }^{[11]}$, applied on an image, is represented as:

$$
\begin{aligned}
\mathrm{G}(\mathrm{x}, \mathrm{y})= & \exp \left(-\Pi\left[\left(\mathrm{x}-\mathrm{x}_{\mathrm{o}}\right)^{2} / \alpha^{2}+\left(\mathrm{y}-\mathrm{y}_{\mathrm{o}}\right)^{2} / \beta^{2}\right]\right) \\
& \exp \left(-2 \pi i\left[\mathrm{u}_{\mathrm{o}}\left(\mathrm{x}-\mathrm{x}_{\mathrm{o}}\right)+\mathrm{v}_{\mathrm{o}}\left(\mathrm{y}-\mathrm{y}_{\mathrm{o}}\right)\right]\right)
\end{aligned}
$$

where $\left(x_{0}, y_{0}\right)$ specify position in the image, $(\alpha, \beta)$ specify the effective width and length, and $\left(u_{0}, v_{o}\right)$ specify modulation, which has spatial frequency $\omega_{0}{ }^{2}=\mathrm{u}_{0}{ }^{2}+\mathrm{v}_{\mathrm{o}}{ }^{2}$.

The phase information in the texture is used because phase angles are assigned on the account of the image contrast. 2D Gabor filter determines in which quadrant the phasor lies using the wavelet. The family of 2-D elementary functions constitutes a generalization of the 1 -D elementary functions proposed by Gabor ${ }^{[12]}$. The real parts of 2D Gabor filters are shorten to be volume zero and get illumination invariance. For each resulting bit, interpret the signs of the real and imaginary parts from quadrature image projections and through quantization assigned binary values: 1 for positive and 0 for negative projection values ${ }^{[13]}$.

\section{Classification}

Classification is the problem of identifying which of the set of categories (sub-populations) a new observation belongs, on the basis of a training set of data containing observations whose category membership is known. For the purpose of matching or classification, various methods are used viz. Hamming Distance ${ }^{[14]}$, Weighted Euclidean Distance, Normalized Correlation, Support Vector Machine (SVM) and Artificial Neural Network (ANN). In this paper, SVM and ANN are used as a classifier for pattern classification to identify individual's identity based on Iris code.

\section{A. Support Vector Machines}

SVM works on principle of structural risk minimization as shown in fig 2. SVM is a binary classifier that separates two classes. Two important aspects for developing SVM as a classifier are determination of the optimal hyperplane which will optimally separate the two classes and the other is transformation of non-linearly separable classification problem into linearly separable problem ${ }^{[15,16]}$. Linearly separable binary classification problem with no possibility of miss-classification data is shown in Fig. 5. Let a set of input feature vector and the class label are $\mathrm{x}$ and $\mathrm{y}$. The input feature vectors and the class label can be represented as $\left\{\mathrm{x}_{\mathrm{i}}, \mathrm{y}_{\mathrm{i}}\right\}$ where $\mathrm{i}=1,2, \ldots, \mathrm{N}$ and $\mathrm{y}= \pm 1$. The separating hyperplane is,

$$
\text { w. } x+b=0
$$

which implies

$$
y_{i}\left(w \cdot x_{i} \cdot b\right)>=1, i=1,2, \ldots N
$$

Basically, there are numerous possible values of $\{\mathbf{w}, \mathbf{b}\}$ that create separating hyperplane. In SVM only hyperplane that maximizes the margin between two sets is used. The optimal hyperplane maximizes the sum of the distances to the closet positive and negative training patterns. The sum is called as margin ${ }^{[17]}$. For non-linear case, training patterns are constructed onto a high dimensional space using kernel functions. Most commonly used kernel functions are polynomial, sigmoid and Gaussian radial basis function. The SVM in general makes four

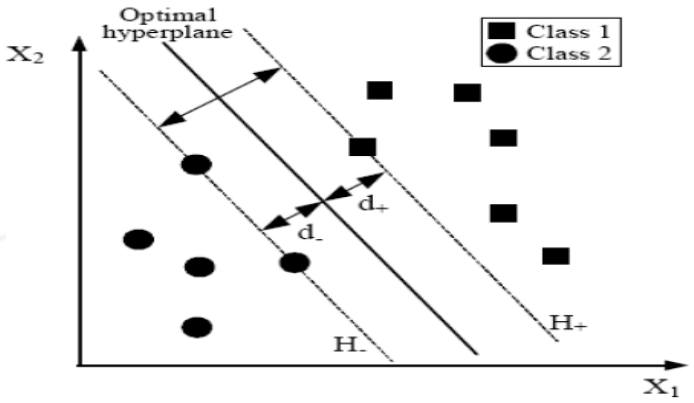

Fig. 6. SVM with linear separable data

possible decision in iris recognition; the authorized person is accepted, the authorized person is rejected, the unauthorized person (impostor) is accepted and the unauthorized person (impostor) is rejected ${ }^{[18]}$

\section{B. Artificial Neural Network}

ANN is a mathematical or computational model that is inspired by the structure and functional aspects of biological neural networks. A neural network is a system of parallel processors connected together as a directed graph. Each neurons of the network is represented as a node ${ }^{[19]}$. ANN is composed of input layer, hidden layer and output layer. ANN has to compare normalized image with original image and identify the individual from the image. In this paper, a feed forward neural network using back propagation (FFBP) algorithm is used for iris pattern classification. The structure of back propagation neural network is shown in Fig. 7.

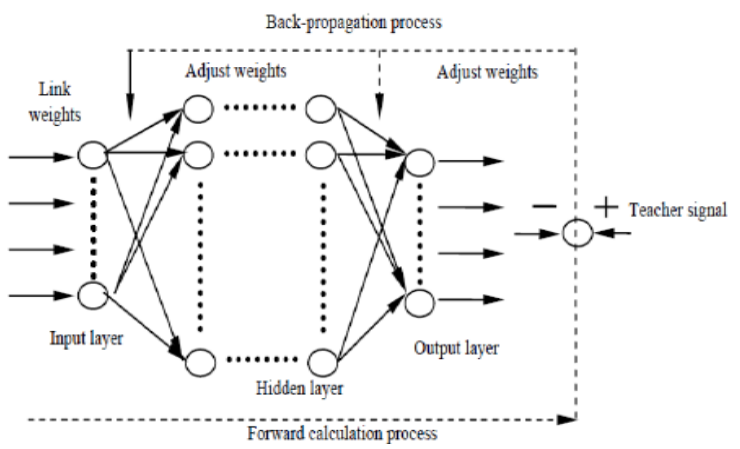

Fig. 7. Stucture of BP neural network

The error back-propagation training algorithm is used to adjust the internal neural network weights. After activation of neural network, the back propagation learning algorithm is applied for training ${ }^{[20]}$. 


\section{VI.RESULTS}

The version The Chinese Academy of Sciences Institute of Automation (CASIA) ${ }^{[21]}$ version 1 eye image database is used in the experiment. CASIA v1 eye image database contains 756 eye images from 108 individuals. All images are stored in BMP format with resolution of $320 * 280$ pixels. Experiments are performing in two steps: iris segmentation and iris recognition. All experiments were performed by using MATLAB version R2007b on core- i3 processor. Iris segmentation process includes pupil- iris boundary localization and iris- sclera boundary. In this paper, iris localization is carried out by means of canny edge detection and circular Hough transform. In localization, eyelashes and eyelids removal perform by parabolic Hough transform. For CASIA v1 database, values for iris radius range from 90 to 150 pixels and pupil radius range from 28 to 75 pixels. Iris normalization is very important to take all iris images in a fixed size because every person has different size of irises. Daugman's rubber sheet model is used for converting iris into polar coordinate system which takes it into a rectangular form. The normalized iris image dimension is of $240 * 20$ pixels. The computer modeling of iris segmentation by canny edge detection and circular Hough transform gives the accuracy rate of $83.92 \%$ in average time of $85 \mathrm{~s}$. 50 person's irises with 5 samples are selected from database for classification. Features of normalized iris are extracted by using 2D Gabor filter which give the texture information. These features are in complex numbers. Two dimension features are then converted into one dimension. 5 samples of each person are segmented and get the total 250 samples of 50 person's iris images. 250 samples and there feature vectors are given to SVM as input on using polynomial kernel of 8. SVM randomly select the testing data. SVM gives the classification rate of $90.25 \%$. On other hand, ANN use back propagation neural network for classification of iris patterns. The training data for ANN is same as SVM and the target has to give to the ANN. ANN gives the accuracy rate of $83.65 \%$ for classification. The accuracy rate of iris segmentation and recognition is given in Table 1. SVM offers a computationally simpler model to obtain the solution which is global minimum and unique, as compared to ANN. Least amount of positive and negative training samples are used for training the SVM classifier as compared to ANN.

Table 1. Accuracy rate of iris segmentation and recognition

\begin{tabular}{|c|c|c|}
\hline Process & Methodology & $\begin{array}{c}\text { Accuracy } \\
\text { Rate (\%) }\end{array}$ \\
\hline Segmentation & Hough Transform & 83.92 \\
\hline \multirow{2}{*}{ Recognition } & $\begin{array}{c}\text { Artificial Neural } \\
\text { Network }\end{array}$ & 83.65 \\
\cline { 2 - 3 } & $\begin{array}{c}\text { Support Vector } \\
\text { Machines }\end{array}$ & 90.25 \\
\hline
\end{tabular}

\section{REFERENCES}

[1] R. Bolle, S. Pankanti and A. Jain, "Biometrics: Personal Identification in a Networked Society", Kluwer, 1999.

[2] R.M. Bolle, J.H. Connel, S. Pankanti, N.K. Ratha and A.W. Senior, Guide to Biometrics, New York: Springer-Verlog, 2003.

[3] B. Miller, "Vital Signs of Identity", IEEE Spectrum, vol. 31, no. 2, pp. 22-30, 1994.

[4] F. Adler, Physiology of the Eye: Clinical Application, Fourth ed. London: The C. V. Mosby Company, 1965.

[5] Li Ma, Tieniu Tan, Yunhong Wang and Dexin Zhang, "Personal Identification Based on Iris Texture Analysis”, IEEE Transactions on Pattern Analysis and Machine Intelligence, vol. 25, no. 12, December 2003.

[6] R. Wildes, "Iris Recognition: An Emerging Biometric Technology", Proceedings of the IEEE, Vol. 85, No. 9, 1997.

[7] Libor Masek, "Recognition of Human Iris Patterns for Biometric Identification", The School of Computer Science and Software Engineering, The University of Western Australia, 2003.

[8] J. Daugman, "High confidence visual recognition of persons by a test of statistical independence", IEEE Trans. Pattern Analysis and Machine Intelligence, vol. 15, pp. 1148-1161, 1993.

[9] A. W.K.Kong, D. Zhang and M.S.Kamel, "An analysis of iris code", IEEE Trans. on Image Processing, vol.-19, pp. 522-532, 2010.

[10] Arun Ross, Manisha Sham Sundar, "Block Based Texture Analysis for Iris Classification and Matching', Proc. of IEEE Computer Society Workshop on Biometrics at the Computer Vision and Pattern Recogniton (CVPR) Conference, (San Francisco, USA), June 2010.

[11] J. Daugman, "Complete Discrete 2-D Gabor Transforms by Neural Networks for Image Analysis and Compression", IEEE TRANSACTIONS ON ACOUSTICS, SPEECH, AND SIGNAL PROCESSING. VOL. 36. NO. 7. JULY 1988.

[12] D. Gabor, “Theory of communication,” J. Inst. Elec. Eng., vol. 93, pp. 429-457, 1946.

[13] H. Proença, L.A. Alexandre, "Iris Segmentation Methodology for Non-cooperative Recognition', IEEE Proceedings of Vision, Image and Signal Processing, pp. 199-205, 2006.

[14] J. Daugman,, "How iris recognition works", Proceedings of 2002 International Conference on Image Processing, Vol. 1, 2002.

[15] C. J. C. Burges, "A tutorial on support vector machines for pattern recognition”. Data Mining and Knowledge Discovery 2(2), 121-167 (1998)

[16] N. Cristianini, T. D. Shawe, "An Introduction to Support Vector Machines and other Kernel-based Learning Methods', Cambridge University press, Cambridge (2000).

[17] B. Son, H. Won, G. Kee and Y. Lee, "Discriminant Iris Feature and Support Vector Machines for Iris Recognition', International Conference on Image Processing, 2004.

[18] Hasimah Ali, Momoh J.E. Salami, Wahyudi, "Iris Recognition System by using Support Vector Machines', International Conference on Computer and Communication Engineering, 2008.

[19] Zeng J, Liu ZQ. Type-2 fuzzy hidden Markov models to phoneme recognition, Proceedings of the International Conference on Pattern Recognition (ICRP), Cambridge 2004: 192-195.

[20] Rahib H. Abiyev and Koray Altunkaya, "Personal Iris Recognition Using Neural Network', International Journal of Security and its Applications, vol. 2, no. 2, April, 2008.

[21] Chinese Academy of Sciences - Institute of Automation. Database of 756 Greyscale Eye Images. http://www.sinobiometrics.com Version 1.0, 2003. 